\title{
Face Recognition through Combined SVD and LBP Features
}

\author{
Rahul Kumar Mittal \\ M.Tech. Scholar \\ BGIET, Sangrur \\ Punjab (India)
}

\author{
Anupam Garg \\ Assistant Professor \\ BGIET, Sangrur \\ Punjab (India)
}

\begin{abstract}
A number of global and local methods are available for the representation of face images, still no single approach is found to be suitable in most of the situations. As the information conveyed by these two feature sets, is different hence the techniques that combine the global and local features together are necessary to obtain the optimal results. In this paper, we have developed an approach to combine two feature sets obtained from SVD and LBP approaches. SVD approach is able to efficiently represent the global variations of face images whereas the LBP is one of the most useful descriptors to extract the local variation of face images. In order to analyse the effectiveness of the proposed approach obtained by the fusion of SVD and LBP approaches, various experiments have been carried out on ORL and Yale face databases. The proposed approach has also been compared to some existing techniques and from the detailed experiments it has been observed that the results obtained by the proposed method are far better than these approaches.
\end{abstract}

\section{Keywords}

Face Recognition, Singular Value decomposition (SVD), Local Binary pattern (LBP), Complementary features, Global Face Descriptors, Local Face Descriptors.

\section{INTRODUCTION}

During past several decades, facial image analysis has received much attention in the area of computer vision and image processing. A lot research is going on in this field because of its wide range of applications such as multimedia, banking, surveillance including criminal identification, security systems, image and film processing, human computer interaction [1]. Due to ample ongoing research in this area, there exist a significant number of feature extraction approaches in literature to represent the face images. These existing face recognition approaches are broadly classified in two categories - global feature extraction approaches and the local feature extraction approaches [2].

These are based on statistical approaches wherein the features are extracted from whole face image. Most frequently used global methods include subspace based methods, spatial frequency techniques and moment based methods. The Eigenface based methods have become the standard and most famous methods in this category are PCA, FLD, LDA, 2DPCA,2D2PCA [3-5]. These global approaches are generally affected by the inclusion of data that are irrelevant to facial portion such as hair, glasses, shoulders and background which may result in creation of erroneous feature vectors that can affect the recognition accuracy of these approaches. Recently, the singular value decomposition (SVD) is used for face recognition in [6, 7]; SVD is a generalization of the eigen-decomposition which can be used to analyze rectangular matrices (the eigen-decomposition is defined only for squared matrices).

The local face recognition methods deal with the interior information included within specific parts of the face images like the features of image patches containing eyes, nose, mouth, cheeks, etc. Recently, lots of work has been done on local feature extraction methods, because they have been proved to be robust against variations in illumination, facial expressions, noise and the occlusion. Among these approaches Local Binary Pattern (LBP) has been recognized as computationally simple and efficient method which is invariant to monotonic gray scale transformation and illumination changes [8-11]. The idea of using LBP for face description is motivated by the fact that faces can be seen as a composition of micro-patterns which are well described by such operator.

In complex applications like face recognition, it is observed that one kind of feature set is not sufficient to capture the whole face information. Thus, finding and combining the complementary feature sets has become an active research topic in recent days [12]. Many such approaches already exist in the literature. Fusion of some selected left and right singular vectors of SVD and DCT-RLDA is capable of generating the superior results on face images as compared to their individual counterpart [13]. Kim et al. [14] have proposed a combined subspace based approach using both global and local features obtained by applying Linear Discriminant Analysis (LDA) based method for face recognition. Fang et al. proposed fusion of global PCA features and Haar wavelet based local features for face verification [15].

In this research work, an approach is developed that combines two feature sets obtained from a global feature extraction and a local feature extraction approach. Global feature extraction technique namely SVD is able to efficiently represent the global variations of face images whereas the selected local feature extraction approach, i.e. LBP is one of the useful descriptors to extract the local variation among face images. In order to analyse the effectiveness of the proposed approach obtained by the fusion of selected global and local approaches, various experiments have been carried out on standard face databases. The recognition accuracy of the proposed approach has also been compared to some existing techniques.

The rest of the paper is organized as follows. Section 2 presents the brief overview of the SVD and the LBP approaches. The practice involved in the proposed fusion of these approaches is described in Section 3. Experiments and the results obtained are presented in Section 4. Conclusions and future directions are presented in Section 5. 


\section{APPROACHES USED IN THIS WORK}

The global and local features demonstrate different characteristics in representing the face images. In general, in case of the global approaches a small size of feature vector is created by extracting the features from the whole image and it is able to depict the common characteristics of the faces. These features must be independent of the image orientation and noise. On the other hand, the features used to represent face images locally should be able to finely extract the variations inside local parts of the faces and should also be independent of image position, scale, size and noise. In view of this, the proposed method includes the SVD approach in order to obtain the global features wherein some selected number of left and right singular vectors is used to create the feature vector. Likewise, the LBP descriptor is able to efficiently extract the local details of face images, i.e. image edges, peaks, etc., even in the presence of noise. The LBP features are invariant to changes in lighting and scale. Therefore, these selected sets of global and local feature vectors are competent to improve the robustness of face recognition system.

\subsection{Singular Value decomposition (SVD)}

Tian et al. suggested that the algebraic features are valid features in object recognition such as face recognition [6]. In their work, only the singular values of SVD method are used in the feature vector. The effectiveness of singular vectors of SVD has been tested in [13]. By analogy with the eigendecomposition which decomposes a matrix into two simple matrices, the main idea of the SVD is to decompose a rectangular matrix into three simple matrices: two orthogonal matrices and one diagonal matrix as follows:

$$
\mathrm{A}=\mathrm{U} \Sigma \mathrm{VT}
$$

where $\quad U=[u 1, u 2, \ldots, u m]$ : left singular vectors

$\mathrm{V}=[\mathrm{v} 1, \mathrm{v} 2, \ldots, \mathrm{vn}]:$ right singular vectors

$\Sigma=\operatorname{diag}(\sigma 1, \sigma 2, \ldots, \sigma p)$ : singular values and $\sigma 1 \geq \sigma 2 \geq \ldots \geq \sigma \mathrm{p}$, $\mathrm{p}=\min (\mathrm{m}, \mathrm{n})$

The columns of the two orthogonal matrices are called the left and right singular vectors and the diagonal matrix contains the singular values. SVD has some important properties such as stability, transposition invariance, rotation invariance, proportion invariance, mirror trans- form invariance. We can classify the SVD-based methods into two categories: those which use singular values as the feature vector [6] and those which use the left and right singular vectors as invariant image features. The authors have observed that using the left and right singular vectors generate superior results in comparison to using the singular values in face recognition[13].

\subsection{Local Binary Pattern (LBP)}

Ojala et al. $[9,10]$ introduced the Local Binary Patterns (LBP) for optimal texture description which is used in many computer vision and pattern recognition applications. The most important property of this approach is its tolerance against illumination variation. The LBP operator assigns a label to every pixel $\mathrm{p}_{\mathrm{i}}$ of an image by thresholding their respective $3 \times 3$ neighborhood values with the value of the central pixel $\mathrm{p}_{\mathrm{c}}$ and produces the result in the form of 8-bit integer binary code as described in Figure 1. The resulting binary pattern is used as a local image descriptor. The LBP operator is computed as

$$
\begin{aligned}
& L B P=\sum_{i=0}^{7} 2^{i} b\left(p_{i}-p_{c}\right) \\
& b\left(p_{i}-p_{c}\right)= \begin{cases}1 & \text { if } p_{i} \geq p_{c} \\
0 & \text { otherwise }\end{cases}
\end{aligned}
$$

wherein the values of $i$ move along the 8-neighbors of the central pixel. The valuable extension to original LBP operator defines the histogram features with uniform patterns (A pattern is said to be uniform if it contains at the most two bitwise transitions from 0 to 1 or vice versa for a circular binary pattern). The binary patterns 10001111 and 11111111 are the examples of uniform patterns. Ojala et al. observed that out of 256 patterns only 58 patterns are uniform and provide approximately $90 \%$ information of the image neighborhoods while the remaining patterns consist of mostly the noise. This attribute significantly reduces the number of LBP histogram bins from 256 to 59 wherein all the nonuniform patterns are stored in a single bin the 59th bin.

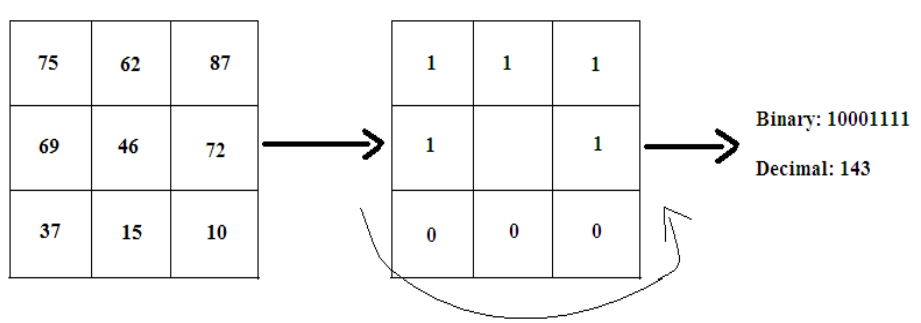

Fig 1: Illustration of basic LBP approach

\subsection{Euclidean distance}

The distance between two feature vectors is computed by using the Euclidean distance which is also known as L2-norm or nearest neighbor classifier. The Euclidean distance between two sets of feature vectors is given by

$$
d_{2}(x, y)=\sqrt{\sum_{k=1}^{L}\left(x_{k}-y_{k}\right)^{2}}
$$

The $\mathrm{x}$ and $\mathrm{y}$ corresponds to the feature vectors of database and the query images, respectively and $L$ is the size of the feature vectors.

\section{THE PROPOSED METHOD}

The research work herein is focused on the development of an approach by combining a global SVD approach and a local LBP approach into a single feature vector. The designed method will comprise of the invariant characteristics of both the approaches therefore it will be able to generate the optimal results against most of the variations (pose, expression and illumination) present in the face images.

The recognition of face images through the proposed fusion of feature sets includes three stages - feature extraction, fusion of similarity score and the classification. The first stage of the procedure creates invariant feature sets extracted by using SVD and LBP approaches. The second stage involves the fusion of feature vectors obtained from these approaches and finally the recognition is performed on the basis of minimum distance evaluated with the help of minimum distance classifier (Euclidean distance). The features of these approaches are normalized before the fusion. The normalization usually maps the values obtained from multiple frameworks onto a common platform. The procedure followed 
to recognize the face images by the proposed combined approach is briefly described in Figure 2 .

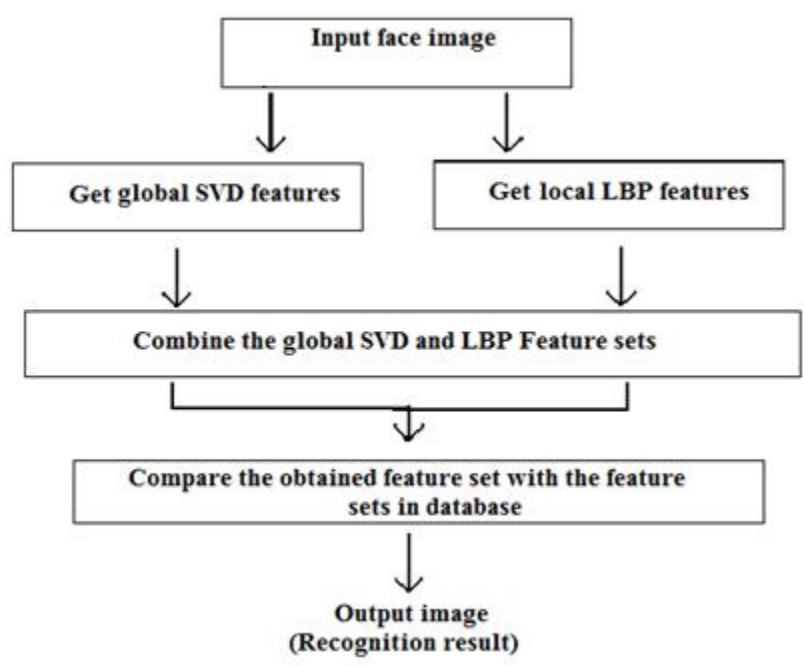

Fig 2: Procedure representing the proposed face recognition method

\section{EXPERIMENTS AND RESULTS}

In order to evaluate the performance of the proposed combined SVD and LBP method various experiments are carried out on ORL and Yale databases by taking different number of images in training and test sets. The performance of the proposed method is compared with individual SVD and LBP approaches as well as it is compared with some recent available methods. For extracting the features, the original images of ORL and Yale databases are cropped to generate the images of size $64 \times 64$ pixels. Some of the sample cropped images from ORL and YALE databases are shown in Figure3.

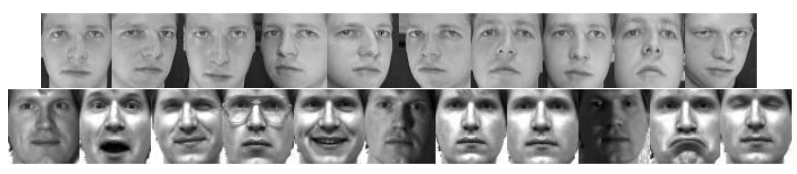

Fig 3: Sample cropped images from ORL and YALE Databases

The experiments are performed on the ORL database with expression, scale and small pose variations. A number of experiments are conducted by extracting the feature vectors for different number of left and right singular vectors, i.e. (r, c), but best results are obtained for the values $(9,7)$ so all the results presented in this paper use these values for the selection of left and right singular vectors. The recognition performance is also affected by the selection of training and test images so the results on a number of differently selected training and test sets are conducted. To evaluate the performance of proposed method against the pose variation the experimental setup designed for the ORL database is as the first set, namely Set1, has first five images (i.e. image number 1, 2, 3, 4 and 5) as training and remaining five as test images. Set 2 consists of even numbered five images (i.e. image number 2, 4, 6, 8 and 10) for training and remaining odd numbered images for testing. Set 3 consists of images selected in the other way, i.e. odd numbered images for training and even numbered for testing. The rest of the sets, i.e. Set 4 and Set 5 randomly select any five images for training and the remaining five are used for testing each set. These setups for ORL database are briefly described in Table 1.
Table 1: Experiment setup for ORL database

\begin{tabular}{|l|l|l|}
\hline $\begin{array}{c}\text { Sr. } \\
\text { No. }\end{array}$ & $\begin{array}{l}\text { Experiment } \\
\text { Setup }\end{array}$ & \multicolumn{1}{c|}{ Description } \\
\hline 1. & Set1 & $\begin{array}{l}\text { First five images of each person are } \\
\text { taken in the training set and } \\
\text { remaining five images are kept in } \\
\text { test set. }\end{array}$ \\
\hline 2 & Set2 & $\begin{array}{l}\text { Even numbered five images of each } \\
\text { person are taken in the training set } \\
\text { and remaining five are kept in the } \\
\text { test set }\end{array}$ \\
\hline 3 & Set3 & $\begin{array}{l}\text { Odd numbered five images of each } \\
\text { person are taken in the training set } \\
\text { and remaining five are kept in the } \\
\text { test set }\end{array}$ \\
\hline 4 & Set4 & $\begin{array}{l}\text { Random five images of each person } \\
\text { are taken in the training set and } \\
\text { remaining all are kept in the test set. }\end{array}$ \\
\hline 5. & Set5 & $\begin{array}{l}\text { Random five images of each person } \\
\text { are taken in the training set and } \\
\text { remaining all are kept in the test set. }\end{array}$ \\
\hline
\end{tabular}

In order to analyze the performance of these approaches across expression and illumination variations exhaustive experiments are performed on Yale database by taking different number of images in training and test sets. For this purpose, similar experiment setup is followed for the face images of this database, i.e. Set1, consists of first five images (i.e. image number 1, 2, 3, 4 and 5) as training and remaining six as test images. Set 2 consists of even numbered five images (i.e. image number $2,4,6,8$ and 10 ) for training and remaining six images for testing. Set3 consists of images selected in the other way, i.e. odd numbered images for training and even numbered for testing. The rest of the sets, i.e. Set 4 and Set 5 randomly select any five images for training and the remaining six images are used for testing each set. This framed setup for Yale database is presented in Table 2.

Table 2: Experiment setup for Yale database

\begin{tabular}{|c|c|l|}
\hline $\begin{array}{c}\text { Sr. } \\
\text { No. }\end{array}$ & $\begin{array}{c}\text { Experiment } \\
\text { Setup }\end{array}$ & \multicolumn{1}{c|}{ Description } \\
\hline 1. & Set1 & $\begin{array}{l}\text { First five images of each person are } \\
\text { taken in the training set and remaining } \\
\text { six images are kept in test set. }\end{array}$ \\
\hline 2 & Set2 & $\begin{array}{l}\text { Even numbered five images of each } \\
\text { person are taken in the training set and } \\
\text { remaining six are kept in the test set }\end{array}$ \\
\hline 3 & Set3 & $\begin{array}{l}\text { Odd numbered five images of each } \\
\text { person are taken in the training set and } \\
\text { remaining six are kept in the test set }\end{array}$ \\
\hline 4 & Set4 & $\begin{array}{l}\text { Random five images of each person } \\
\text { are taken in the training set and } \\
\text { remaining all (six face images) are } \\
\text { kept in the test set. }\end{array}$ \\
\hline 5. & Set5 & $\begin{array}{l}\text { Random five images of each person } \\
\text { are taken in the training set and } \\
\text { remaining all (six face images) are } \\
\text { kept in the test set. }\end{array}$ \\
\hline
\end{tabular}

\subsection{Results on ORL database}

In depth experiments are performed on various testing sets framed and as discussed in the previous experiment setup section. On ORL database, the experiments are performed by 
taking selected five images in the training set and the remaining five images are kept in the test set. In order to impartially analyse the performance of the proposed method the experiments are performed on various sets of training and test images as described in Table 1. In following part of this section we have described the results obtained on these sets against pose and scale variations present in the face images. Various experiments performed on this database and the obtained results are shown in Table 3 .

Table 3: Recognition results on ORL database

\begin{tabular}{|c|c|c|c|}
\hline $\begin{array}{c}\text { Experiment } \\
\text { performed On }\end{array}$ & SVD & LBP & $\begin{array}{c}\text { Proposed } \\
\text { method }\end{array}$ \\
\hline Set1 & 94.5 & 92.5 & 97.0 \\
\hline Set2 & 97.0 & 96.0 & 99.5 \\
\hline Set3 & 97.5 & 96.5 & 99.0 \\
\hline Set4 & 97.0 & 96.0 & 99.5 \\
\hline Set5 & 98.0 & 97.5 & 99.0 \\
\hline
\end{tabular}

It is clear from the experiments carried out on all the sets of training and test images that the recognition rate obtained by the proposed method is superior to that obtained by the individual SVD and LBP approaches. For example on set1 a recognition rate of $94.5 \%$ is achieved by the SVD and a recognition rate of $92.5 \%$ is obtained with LBP approach whereas the recognition rate achieved by the proposed method is $97.0 \%$. Amongst the individual approaches, SVD performs better on this database as compared to the LBP approach.

\subsection{Results on Yale database}

Further, experiments on various sets of training and test images (shown in Table 2) are done on Yale database in order to analyse the performance of the proposed method against expression and illumination variation. The results obtained for this experimental setup are shown in Table 4. It is observed from the obtained results that the performance of the proposed method is significantly higher than that of the individual approaches which proves the robustness of the proposed method over changes in expression and illumination. As it is well known that LBP is one of the best approaches for illumination variation, so the results obtained the LBP approach are far better than that of the SVD approach. For example on set 1 the recognition rate obtained with SVD is only $72.2 \%$ whereas the LBP approach attained a recognition rate of $92.2 \%$. However on this set, the recognition rate achieved by the proposed method is $95.5 \%$ which is significantly higher than that obtained by both of the individual SVD and LBP approaches.

Table 4: Recognition results on Yale database

\begin{tabular}{|c|c|c|c|}
\hline $\begin{array}{c}\text { Experiment } \\
\text { performed On }\end{array}$ & SVD & LBP & $\begin{array}{c}\text { Proposed } \\
\text { method }\end{array}$ \\
\hline Set1 & 72.2 & 92.2 & 95.5 \\
\hline Set2 & 72.2 & 95.5 & 95.56 \\
\hline Set3 & 77.8 & 96.7 & 97.7 \\
\hline Set4 & 81.1 & 95.56 & 96.67 \\
\hline Set5 & 78.89 & 94.4 & 96.67 \\
\hline
\end{tabular}

\subsection{Performance Comparison}

In this part, the performance of the proposed method is analysed against some of the recent available methods. Several LBP based and eigenface methods are tested in order to demonstrate the superiority of the proposed approach. Table 5 shows the recognition accuracy of some latest and well known face recognition methods on Yale and on ORL databases wherein first five images of each person are taken in the training set and remaining all are kept in the test set, i.e. set1. Similarly for Yale dataset first five images of each person are taken for training and remaining all are used in the test set. From the presented results, it is observed that, on ORL and Yale databases, the recognition accuracy of the proposed method is better than all the approaches mentioned in the table.

Table 5: Performance comparison of various face recognition approaches

\begin{tabular}{|l|c|c|}
\hline \multirow{2}{*}{ Method } & \multicolumn{2}{c|}{$\begin{array}{c}\text { Recognition rate in } \\
\text { percentage }\end{array}$} \\
\cline { 2 - 3 } & ORL & YALE \\
\hline PCA (Eigenface) [17] & 87.8 & 85.7 \\
\hline LDA [16] & 91.0 & 81.89 \\
\hline 2D-LDA [16] & 92.5 & 86.57 \\
\hline 2D-WLDA [16] & 93.5 & 88.0 \\
\hline 2D-DWLDA [16] & 94.0 & 89.33 \\
\hline SVD & 98.0 & 81.1 \\
\hline LBP & 97.5 & 95.56 \\
\hline Proposed method & 99.0 & 96.67 \\
\hline
\end{tabular}

\section{CONCLUSION}

In this research work, an approach to combine the global and local features is presented in order to obtain the benefits of both of these. The proposed approach uses the SVD and LBP approaches to obtain the respective global and local features from the face images. The effectiveness of the proposed approach, obtained by the fusion of SVD and LBP approaches, is evaluated through various experiments carried out on two standard face databases. In order to perform detailed experiments a number of training and test sets are framed for both ORL and Yale databases. From the experiments carried out on these formulated sets of training and test images, it is observed that the recognition rate obtained by the proposed method is superior to that obtained by the individual SVD and LBP approaches. The recognition accuracy of the proposed approach has also been compared to some existing techniques and it is observed that on these databases the recognition accuracy of the proposed method is better than the accuracy of these well-known face recognition methods. The future work will include the formation of improved techniques to combine the global and local feature sets.

\section{REFERENCES}

[1] Zhao, W., Chellappa, R., Rosenfeld, A. and Phillips, P. 2003. Face recognition: A literature survey. ACM Computing Surveys, 399-458.

[2] Tolba, A. S., El-Baz, A. H. and El-Harby, A.A. 2006. Face Recognition: A Literature Review. International Journal of Signal Processing 2 (2), 80-103.

[3] Belhumeur, P. N., Hespanha J. P. and Kriegman, D. J. 1997. Eigenfaces vs. Fisherfaces: Recognition using class specific linear projection. IEEE Transactions on Pattern Analysis and Machine Intelligence. 19 (7), 711-720.

[4] Xu, Y., Zhang, D., Yang J. and Yang, J. -Y. 2008. An approach for directly extracting features from matrix data and its application in face recognition. Neurocomputing 71, 1857-1865. 
[5] Daoqiang Z. and Zhi-Hua, Z. 2005. (2D) ${ }^{2}$ PCA: Two directional two dimensional PCA for efficient face representation and recognition. Neurocomputing 69, 224-231.

[6] Tian, Y., Tan, T., Wang, Y. and Fang, Y. 2003. Do singular values contain adequate information for face recognition?. Pattern Recognition 36, 649-655.

[7] Hsu, C-H. and Chen, C-C. 2007. SVD-Based Projection for Face Recognition. In Proceedings of IEEE EIT 2007, 703-706.

[8] $\mathrm{Pu}, \mathrm{X}-\mathrm{R}$., Zhou, Yi. and Zhou, R-Y. 2012. Face Recognition on Partial and Holistic LBP Features. Journal of Electronic Science And Technology 10 (1), $56-60$.

[9] Ahonen, T., Hadid A. and Pietika inen, M. 2006. Face Description with Local Binary Patterns: Application to Face Recognition. IEEE Transactions on Pattern Analysis and Machine Intelligence 28 (12), 2037-2041.

[10] T. Ojala, Matti Pietikäinen and Topi Mäenpää, Gray Scale and Rotation Invariant Texture Classification with Local Binary Patterns, IEEE Transactions on Pattern Analysis and Machine Intelligence, 24 (7), 2002, 971-987.

[11] Huang, D., Shan, C., Ardabilian, M., Wang Y. and Chen, L. 2011. Local Binary Patterns and Its Application to Facial Image Analysis: A Survey. IEEE Transactions on
Systems, Man and Cybernetics-Part C: Applications and Reviews 41(6), 765-781.

[12] Heisele, B., Wu P. H. J. and Poggio, T. 2003. Face recognition: component-based versus global approaches, Computer Vision and Image Understanding 91, 6-21.

[13] Bengherabi, V., Mezai, L., Harizi, F., Guessoum, A. and Cheriet, M. 2008. Score Fusion of SVD and DCT-RLDA for Face Recognition. In Proceedings of IEEE International Conference on Image Processing theory, Tools and Applications, 1-8.

[14] Kim, J. Oh and Choi, C. 2007. Combined Subspace Method Using Global and Local features For Face Recognition. In Proceedings of the IEEE International Joint Conference on Neural Networks (4), 2030-2035.

[15] Fang, Y., Tan, T. and Wang, Y. 2002. Fusion of Global and Local Features for Face Verification. In Proceedings of the IEEE conference on Pattern Recognition, 382-385.

[16] Zhou, D., Yang, X., Peng, N. and Wang, Y. 2006. Improved-LDA based face recognition using both facial global and local information. Pattern Recognition Letters, 536-543.

[17] Zhi, R. and Ruan, Q. 2008. Two-dimensional direct and weighted linear discriminant analysis for face recognition. Neurocomputing, 3607-3611. 